\title{
Internet-based integration for collaborative business processes: implementing the technology in SMEs
}

\author{
Marco Macchi $i^{(*)}$, Marco Garetti ${ }^{(*)}$, Paolo Locatelli ${ }^{(* *)}$, Renata Diazzi $^{(* * *)}$ \\ (*) Politecnico di Milano, Dipartimento di Ingegneria Gestionale, Piazza Leonardo da Vinci, \\ 32, 20133, Milano, Italy. \\ ${ }^{(* *)}$ Consorzio Politecnico Innovazione, EBlab, via Durando, 10, 20158, Milano, Italy. \\ ${ }^{(* * *)}$ Consorzio Politecnico Innovazione, via Fucini, 2, 20133 Milano
}

\begin{abstract}
The present paper aims to discuss the principles of the Internet-based integration of business processes for inter-enterprise collaboration. The Internet-based integration is specifically concerned with the "heterogeneous" context of SMEs, where a broad range of "heterogeneous" tools are involved in the local operation of the business processes at each partner site. The paper discusses the adoption of a "neutral" ("generic") modelling approach as a key principle to achieve integration of "heterogeneity" in the Internet facility. Starting from the "neutral" model proposed for integration, afterwards, the paper introduces the adoption of mechanisms of dynamic configuration of the Internet site views operated by each business partner, thus, preventing from a "generic" operation of the Internet facility. The integration principles are discussed based on the results of an actually on-going project which aims to develop an Internet portal to support collaborative business processes amongst SMEs for the electronic and mechanical sectors.
\end{abstract}

\author{
Keywords \\ Collaborative Business Processes, Internet-based integration, Supply Chain Management, \\ SMEs.
}

\section{INTRODUCTION}

The industrial context of the recent years has been characterized by an ever growing relevance of the outsourcing of the manufacturing, planning and design activities. The ever growing importance of industrial relationships in networks of enterprises is a direct consequence of the specialization of the competencies derived from those outsourcing trends. Moreover, the collaboration within networks of enterprises has correspondingly become one of the most relevant factors to enable the achievement and improvement of their competitiveness.

In this context, the Internet evolution is actually offering new technological opportunities to aid and improve inter-enterprise collaboration, by providing the technological infrastructure to facilitate inter-enterprise integration. In fact, in the late 90ies, the rapid growth of the Internet and its increasing efficiency, reliability 
and safeness in data transfer were the main technological issues facilitating the emergence of the electronic commerce and of the Internet-based integration of companies. To this regard, a strong penetration of the Internet was registered mostly amongst U.S. and North European users. In Italy, the Internet penetration was lower. However, it registered a relevant acceleration from 1998 to 1999 , when the Internet users grew up from 540000 users up to 3,7 millions in the consumers market and from 250000 users to 1,5 millions in the business market (with a noticeable $41 \%$ of SMEs (source ANFOV from [2]). The most part of these Internet applications were however limited to some specific functions, mainly concerned with buying relationships and the integration of commercial processes.

Nowadays, the Internet growth has lead to plenty of proposals for business models of e-commerce both for Business to Consumer (B2C) and for Business to Business (B2B) relationships ([10]). Referring, in particular, to the $B 2 B$ models and to the supply chain processes, a relevant interest is actually noticeable for the Internet-based integration of product and process development, for the supply and manufacturing planning activities, for actuating co-design, co-planning and comanufacturing strategies. This trend is largely demonstrated also by the many Internet-based solutions proposed by all the main software vendors, both for the supply collaborative planning (e.g., Oracle, SAP, Baan) and for the product and process co-design (e.g., EDS PLM solutions).

The present paper discusses a $B 2 B$ model for the Internet-based integration of suppliers with their contractor along a supply chain for collaborative planning and manufacturing, in the special context of SMEs. As well known, SMEs are in Italy a strong reality supporting in a noticeable way the overall economy [1], [4], [6]. Their main operation is particularly relevant in distributed and collaborative production environments, wherein the outsourcing of design, engineering and production processes leads to the need of a continuous interchange of information amongst business partners. This is the reason why the Internet facility is very important for those sectors where a lot of SMEs collaborate combined in multiple clusters.

In the model, that the paper deals with, each enterprise operates autonomously its business processes-supported by its own tools-and collaborates with the other network partners by means of the Internet integrating facility (Figure 1). 


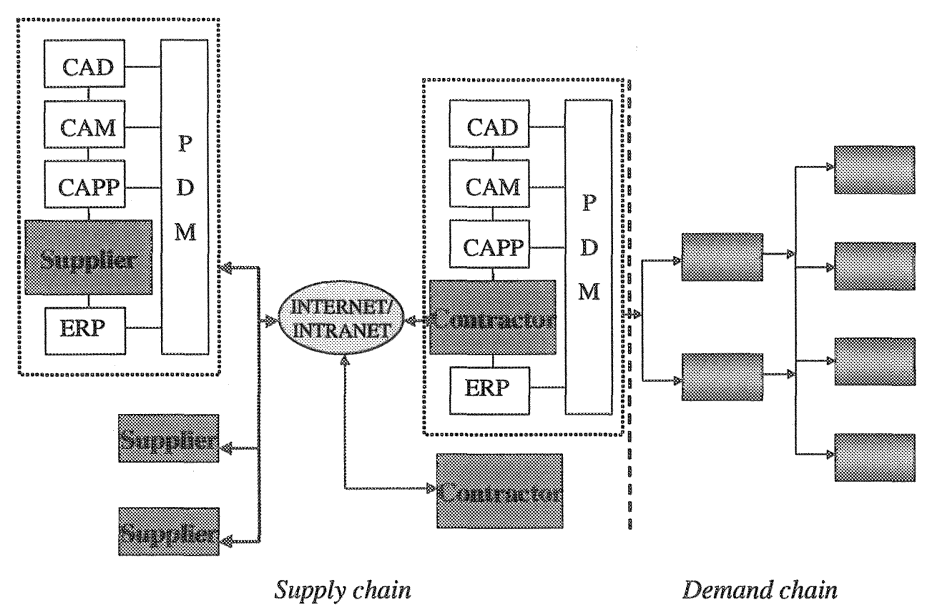

Figure 1 - B2B model for an Internet-based integration of a supply chain of manufacturing collaborative partners (adapted from [10])

In particular, the contractor exchanges, via the Internet and with each of its suppliers, the supply data (orders and stock information) and the related technical and quality documentation. Due to the considered context (a lot of different SMEs cooperating in ever changing clusters), supply and documentation data are produced by means of "heterogeneous" application tools at each partner site, thus setting a problem of "heterogeneous" context integration.

The paper points out the main issues and challenges involved in the integration of such an "heterogeneous" context. In particular, a "neutral" modelling approach is proposed to enable the Internet-based integration. The industrial case studies presented along the paper aims to demonstrate the applicability of such a "neutral" approach. The demonstration aims then to provide evidence that, whilst the design is almost "neutral" ("generic"), to deal with the "heterogeneity" of tools, the design itself, by means of dynamic configuration mechanisms, is capable to meet the "customization" of the site views required by small-sized enterprises.

The paper is correspondingly organized. After a general perspective on collaborative manufacturing models - that the paper is specifically concerned with and their applicability for Internet-based integration (par. 2), the "neutral" modelling approach is presented (par. 3). Needs for "customization" are, then, described based on two industrial clusters in the electronic sector (par. 4). Afterwards demonstration is provided (par. 5): the IT architecture, developed starting from the "neutral" design, is described in its main design issues; demonstration of its compliance with the "customization" challenge is provided. Eventually (par. 6), the integration architecture is discussed against benefits underpinned its adoption in the SMEs industrial contexts and the necessary conditions enabling real utilization during daily operations. 


\section{INTEGRATION FOR COLLABORATIVE MANUTACTURING}

Internet-based integration for collaborative manufacturing in SMEs mainly concerns supply chain management (collaborative planning is actuated via the Internet) and exchange of technical and quality documentation (product and process specifications and quality documentation are exchanged via the Internet). The following table identifies the exchanged information and the business processes falling within the scope of integration.

Table 1 - Business processes and information integrated by the Internet facility

\begin{tabular}{|c|c|c|c|}
\hline & $\begin{array}{c}\text { Supply } \\
\text { data }\end{array}$ & $\begin{array}{c}\text { Technical } \\
\text { documentation }\end{array}$ & $\begin{array}{c}\text { Quality } \\
\text { documentation }\end{array}$ \\
\hline $\begin{array}{l}\text { loventory } \\
\text { management }\end{array}$ & $\begin{array}{l}\text { Stock existence, } \\
\text { inventory control } \\
\text { parameters, } \\
\text { inventory } \\
\text { replenishment }\end{array}$ & $\ldots$ & $\cdots$ \\
\hline $\begin{array}{l}\text { Order } \\
\text { Fulfillment }\end{array}$ & $\begin{array}{l}\text { Order release, } \\
\text { expected order } \\
\text { delivery, order } \\
\text { shipment } \\
\end{array}$ & $\cdots$ & $\cdots$ \\
\hline $\begin{array}{l}\text { Specification } \\
\text { Release }\end{array}$ & $\cdots$ & $\begin{array}{l}\text { Product } \\
\text { specification, } \\
\text { component } \\
\text { specification, process } \\
\text { specification }\end{array}$ & $\cdots$ \\
\hline $\begin{array}{l}\text { Periodic } \\
\text { quality reporting }\end{array}$ & $\cdots$ & $\cdots$ & $\begin{array}{l}\text { Periodic quality } \\
\text { reports, on - } \\
\text { condition quality } \\
\text { reports }\end{array}$ \\
\hline $\begin{array}{l}\text { Lot linspection } \\
\text { reporting }\end{array}$ & $\cdots$ & $\cdots$ & $\begin{array}{l}\text { Lot inspection } \\
\text { reports }\end{array}$ \\
\hline
\end{tabular}

The following sections provide an overview of the corresponding functionalities. The collaborative planning functionalities will be, at first, illustrated. The quality and technical cooperation functionalities will be presented afterwards.

\section{Internet-based integration of vendor managed inventory}

The Vendor Managed Inventory (VMI) model [9] is applicable via the Internet and, in fact, it requires informative facilities to enable the information exchange amongst a supplier and a client. This is closely related to the VMI organizational model. In this model, traditional responsibilities, of the supply relationship, are changed. The client abstains from sending orders to its suppliers. It, instead, installs stocks, whose materials are owned by the suppliers themselves until picking time. It is then the responsibility of the supplier to supply materials according to predefined inventory control parameters. Inventory control parameters are, however, typically defined after a coordinated decision amongst the client and the supplier, aiming, on 
one hand, at achievement of a proper service level, on the other hand, of costs optimization.

The VMI actuation in the Internet facility requires provision of information exchange services to support the inventory visibility of actual stock existences at client production site and the communication of inventory control parameters. In particular, visibility on the client resident inventory is necessary to allow the supplier to control simultaneously both his own inventory and his client's inventory and, thereafter, plan with respect to a system-wide inventory. Besides, to improve its system-wide view, also demand data may be typically transmitted from the client to the supplier.

\section{Intermet-based integration of inventory replenishment}

Beside the VMI model, also traditional inventory management models are applicable in the Internet environment.

Unlike the VMI model, however, in this case orders are released to replenish the inventory. It is in fact the client that is responsible to release orders. Differently from the VMI, then, replenishment orders are sent to the suppliers. In particular, order release time-lags depend on the adopted inventory review system: in case of periodic review system, the inventory level is reviewed and orders are released every periodic time-lag; in case of continuous review, orders are released at variant time-lags, i.e. if the inventory level falls below a reorder point.

The actuation of the traditional inventory management models in the Internet facility requires provision of information exchange services to support the replenishment communications.

\section{Intermet-based integration of order fullilment}

The order fulfilment process includes all the activities ranging from order release to order shipment, including also procurement of raw materials and other components. The order release, held by the client to issue a supply order, and the order shipment, communicated by the supplier to anticipate the actual order delivery, are applicable via the Internet. Referring to the order release, in particular, the order release may also require the communication from the supplier of the expected order delivery time. Besides, also the procurement communication is typically applied in the Internet (see the e-marketplace). Procurement is, however, beyond the scope of this paper.

Thus, to the paper concern, the order fulfilment actuation in Internet requires provision of information exchange services to communicate order releases, expected delivery times and order shipments.

\section{Internet-based integration of the quality and technical documentation exchange}

The exchange of technical and quality documentation includes issuing the respective documentation in the Internet and subsequent document consultation. The 
product and process specifications are issued only when their final release is achieved, after the supplier commits to produce according to these final specifications. The exchange of technical documentation by the Internet aims then to facilitate effective product-process changes (ease transitions from older to newer specifications) to start new production activities in the supplier site. Co-design activities are outside the scope of integration. The quality documentation is exchanged for monitoring sake. The monitoring activities regard the quality parameters both in medium term periods (quality parameters are collected by means of periodic quality reports and, eventually, on-condition reports when the parameters themselves are getting worst on a medium term basis) and short term periods (this is the case of a lot inspection report issued when unacceptable quality parameters are measured for a single lot).

The exchange of quality and technical documentation requires the provision of documentation exchange services to load the documentation in organized archives and, afterwards, to facilitate effective search activities out of the archives themselves. Moreover, an important functionality is the document notification. This is required, on one hand, to notify that new documents are issued on the Internet, on the other hand, to control that each document has been received and accepted. This is very important, in particular, for new product and process specifications.

\section{THE BUSINESS ARCHITECTURE}

\section{The methodological approach}

This section concerns the presentation of the business architecture adopted to integrate a "generic" network of enterprises composed of a contractor and its suppliers. It concerns both a data model - to structure the intra-cluster information exchanges - and a work flow model - to structure the exchange dynamics, developed in ARIS [8], [5]. The E/R method is adopted to define the data model: it defines the information entities pertaining to the Internet-based information exchanges, their information attributes and relations. The UML sequence diagrams are then used to describe the work flow model: they represent the sequences of activities to enable the Internet-based information exchanges.

The architecture has been built based on the framework presented in table 1 . Starting from this framework, the authors propose a "neutral" model to actuate the information exchanges that it is concerned with. From now on, the paper mainly discusses the "neutral" model for the supply data. In particular, as cleared out in the next sections, within the "neutral" model, a special concern is given to the distinction between its really "neutral" (i.e., really "generic") elements and elements characterized with some level of "specificity". This helps to point out the basic cornerstones of the "neutral" model against the elements that may be subject to further "specialization" to respond to the "particular" needs of a specific network of enterprises. Thereafter (in par. 4), two industrial clusters of SMEs of the electronic industry are adopted to evaluate the "neutral" modelling approach against their "customization" requirements. 


\section{The "neutral" data model}

The following Figure provides a partial view of the overall data model proposed for the two SMEs networks considered in this study. It encompasses the data required to actuate both the VMI and the inventory replenishment processes. It helps to describe the "neutral" design approach that the authors adopted for the Internetbased integration.

The "neutral" model is designed around the "Inventory" entity and its related entities ("Stock", "Product" and "Supplier"). In the authors' intention, these entities are the cornerstones of the "neutral" model for the Internet-based cooperation in inventory management processes. From a "generic" perspective, in fact, "inventory" is always associated with a "product", the "supplier" responsible for its replenishment and the "stock" installation wherein the product is physically available. Thereafter, the related key attributes (supplier, product and stock codes) appear to be the really "neutral" ("generic") information.

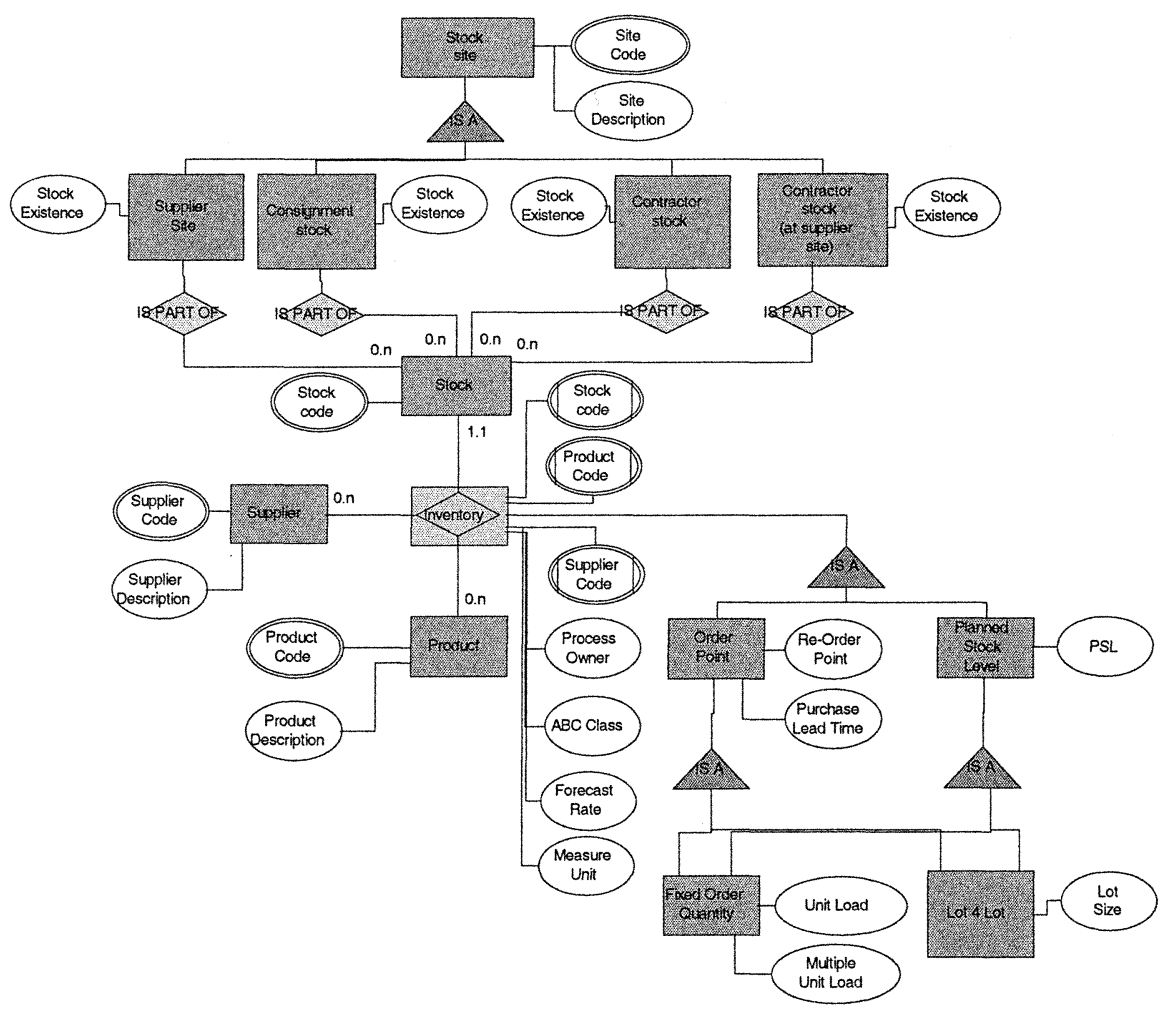

Figure 2 - "Neutral" data model for the VMI and replenishment processes (in a "generic" cluster)

Conversely, even though it is defined from a general perspective, the other information of the $\mathrm{E} / \mathrm{R}$ model have, more or less, characters of "specificity" and may be subject to changes depending on the specific network of enterprises. The following examples can demonstrate the above concept. 
" The "inventory" is defined in the $E / R$ with plenty of parameters. Those parameters are however not a comprehensive list. In fact, they can be considered as defined for a "specific" network of enterprises: they may be either exchanged or not depending on the business processes locally operated by the network partners. As an example, the $A B C$ class attribute may be mentioned: it is exchanged only when the contractor adopts an $\mathrm{ABC}$ classification of products.

" The "inventory" is also defined according to different control policies potentially applicable via the Internet, including the most common ones (either a "re - order point" or a "planned stock level" policy combined with a "fixed order quantity" or a "lot for lot" policy). It is however built with "specific" reference to one of the two clusters considered in the study and includes the information exchanges required in this cluster. It might require further specifications depending on the policies and information exchanges in other clusters. For example, in this cluster, the "planned stock level" policy includes only one stock level (one "PSL"), it might be extended with different stock levels when different replenishment urgencies are implemented.

" Eventually, as said, from a "generic" perspective, the "inventory" is also associated with its physical "stock". However, different types of stock can be identified. The stock types, identified in the $E / R$ model, are "specific" themselves. E.g., the consignment stock is a stock existence installed at the client production site, whilst the material is owned by the supplier until picking time. This stock type is present when such a logistic policy is adopted within the contractor - supplier relationship.

Such a "neutral" modelling approach has been similarly applied to the order fulfilment process and its information exchanges. Therefore, an E/R model has been built to enable the data transfer for order release, order shipment and expected order delivery. Likewise, within this model, it has been possible to identify its really "neutral" elements (i.e., the "product", the "supplier" entities and their "order entry" relationships) and the elements characterized by some level of "specificity" (e.g. different types of "order entry").

As a general conclusion for the "neutral" data modelling approach adopted, we can conclude that:

" the overall E/R model was designed as "generic" as possible, i.e. as a superset of potential information exchanges, derived from a review of inventory management and order fulfilment processes;

- the "generic" model is the conceptual basis to implement a "neutral" data base supporting the integration process and capable to cover, as a whole information structure, partial data needs arising from different collaborative processes;

- it should be observed, however, that there are two types of information structures in the "neutral" data base: some information structures are fixed, being them really "generic" data elements; other information structures may potentially require some "specialization" according to the actual operation of the network partners (and their different collaborative processes). 
The really "neutral" data elements are therefore the cornerstones of the "neutral" data base (see par. 5).

\section{The "neutral" work llows}

The data elements are transmitted through the Internet facility by work flows (and related functions). Correspondingly with the data, also the work flow design may then concern both "neutral" functions (i.e., the primitive functions capable to transfer the minimal set of "neutral" data elements) and extended functions (i.e., functions enabling, based upon the minimal transfer capability, the transfer of the "specific" data flows). The following Figure represents the operation of two work flows actuating the data transfer for order fulfilment and can help demonstrating the above concept.

The first scenario concerns order release and order shipment communications (the order shipment is also anticipated by the announcement of in-transit material, issued after the stock existence is increased in the storage plant and it is ready for delivery). The second scenario is more complex. During the production throughput time, the communication is held, at first, to announce the expected order delivery time, afterwards, to communicate the actual order shipment (split in more order shipments).

The functions, required by the two work flows (such as, e.g., the "Release Order ()", the "Load Order ()", the "Communicate Order Shipment (lot1)", etc), are, at a "generic" design level, structured to operate as "neutral" functions - i.e. to operate on "neutral" data items - (they transfer information by operating only on "product", "supply" and "order entry" items). The same applies to the work flows for inventory data transfer. The only difference is that the related functions operate only on corresponding "neutral" data items ("product", "supply", "inventory" and "stock" items). In the work flows shown in the previous Figure, the "update in-transit material" is an example of those kind of functions. On the other hand, whenever "specific" information flows require to be actuated, "specific" functions may be derived from their "neutral" counterparts. These "specific" functions inherit their "generic" functionality - they always operate on the "neutral" data items - . They extend the "generic" functionality to enable the "specific" flows concerned within the data transfer.

As a general conclusion, according to the work flow design, the "neutral" functions have been fixed once for the Internet portal; their extended functions are to be developed in a customized fashion, depending on the "specific" information flows transferred. 

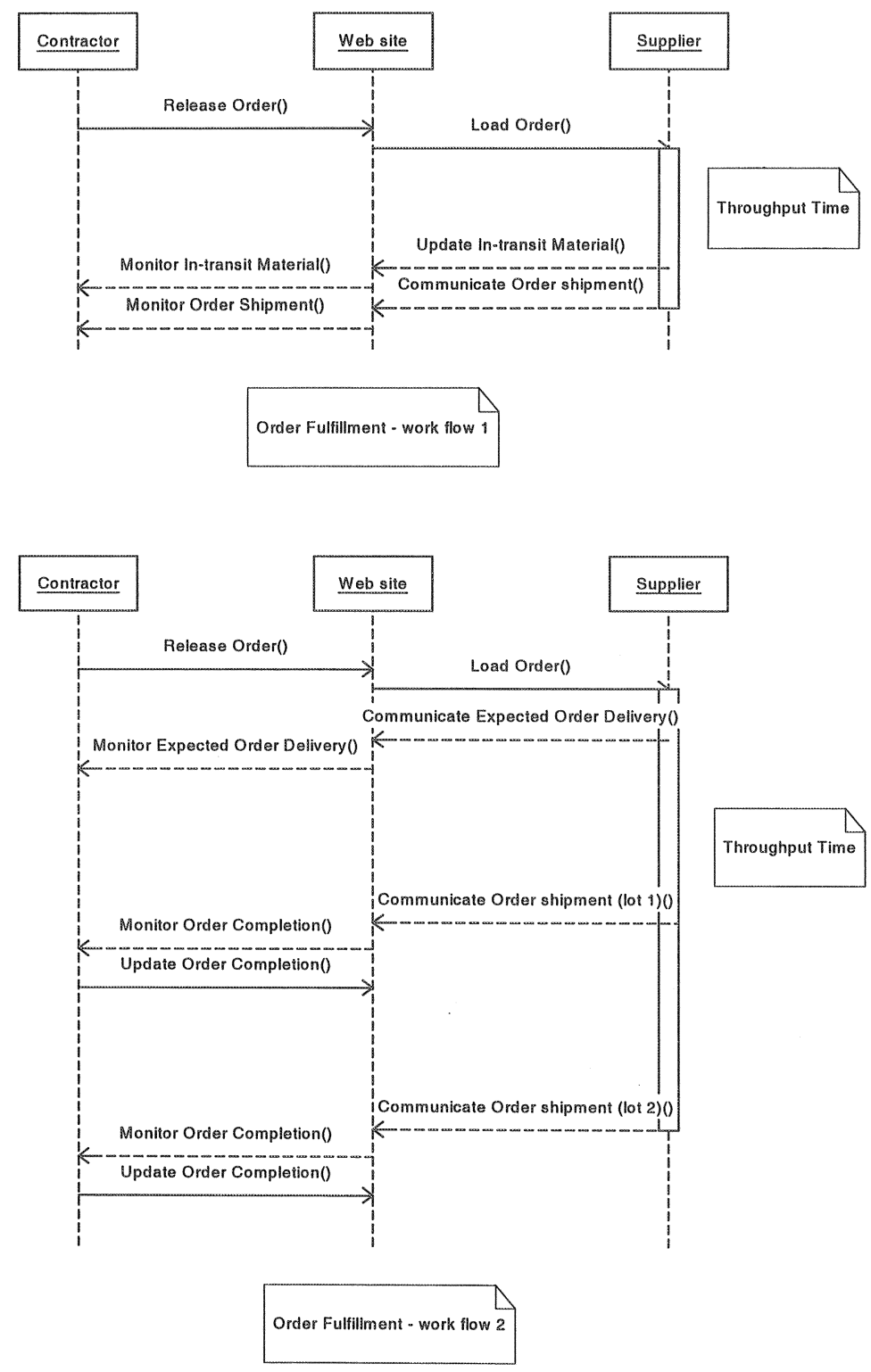

Figure 3 - Order fulfilment process and alternative work flows 


\section{THE CASE STUDY}

\section{Network Organization}

The case study regards two industrial clusters of the electronic industry. In the first cluster, the contractor, producer of electronic instruments, collaborates with its assembly suppliers: instruments are delivered by each supplier to the contractor ready for distribution to the final clients; at most, the contractor acts as quality controller of the supplied products by means of sample testing activities. In the second cluster, the contractor produces industrial PCs and LCD monitors for control panels and collaborates with electronic board assemblers: electronic boards are delivered by suppliers and they are assembled in final products by the contractor.

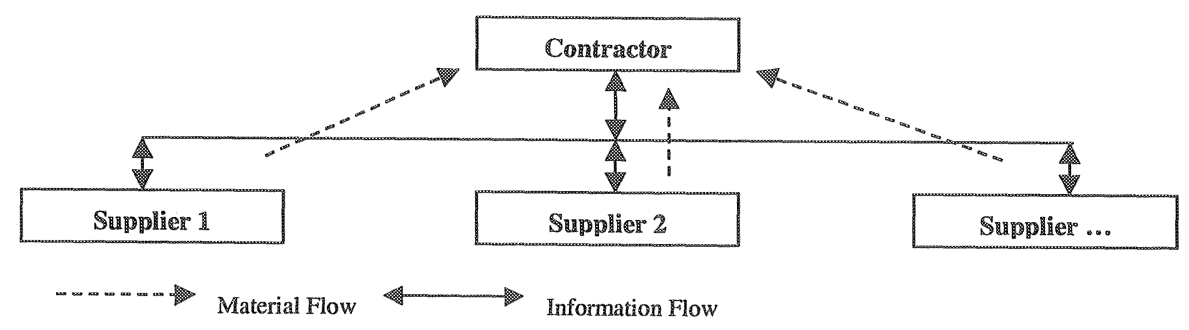

Figure 4 - Cluster organization structure

\section{User needs}

While discussing user needs within the cluster environments, a significant need to ascertain the potentiality of Internet-based collaboration emerged mostly from contractors. Suppliers, mostly very small-sized suppliers, were not so eager to adopt the Internet, fearing a relevant change in their working daily routine. Contractors, in fact, required the establishment of Internet-based relationships to facilitate frequent and standardised information exchanges within the cluster of suppliers. Conversely, small-sized suppliers were interpreting the adoption of the Internet mostly as a constraint imposed by their contractors. However, the analysis of clusters allowed to generalize two integration requirements almost shared amongst contractors and suppliers.

a Integration of information transactions (Figure 5) rather than integration of decision-making processes (and tools) was required. In fact, decision-making was not concerned for integration: the preservation of local autonomy of cluster members and the "heterogeneity" of their operations were key issues of the integration approach.

- Accordingly, the Internet was interpreted mostly as an infrastructure to extend the available information. To this end, proper views of information (resident in the web archive) were strongly required. 


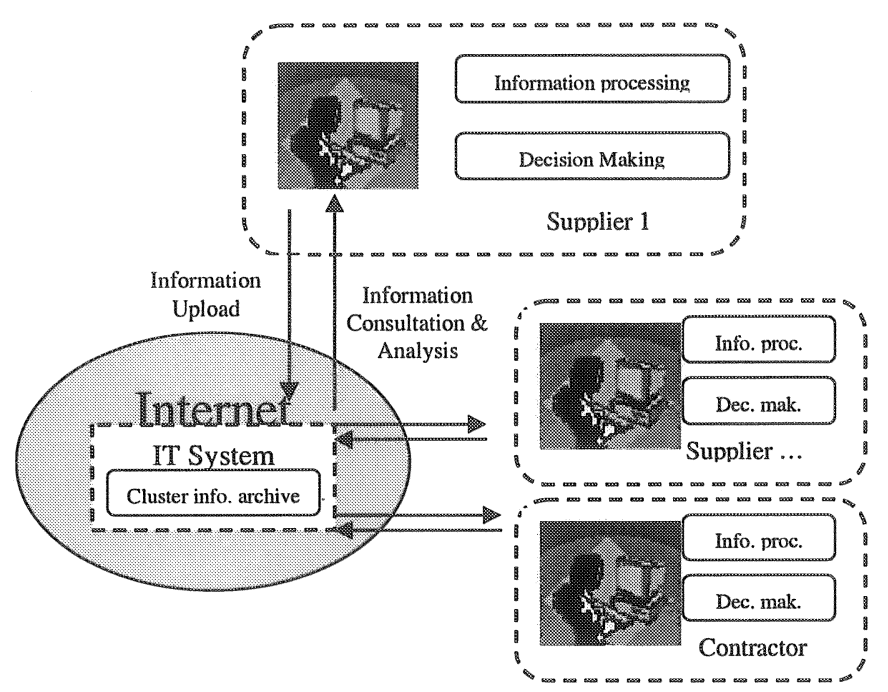

Figure 5 - Integration requirements for Internet adoption: architecture

In the authors" opinion, "customization" is particularly relevant to facilitate Internet adoption during the daily operations both for the contractors and the suppliers. Especially, a "graceful" introduction of the Internet facility is strongly required for small-sized enterprises: if the adoption of Internet would require relevant changes in their work flow operation, a relevant risk of failure would exist. Next section will therefore illustrate the IT architecture adopted to answer "customization" needs in "heterogeneous" networks.

\section{THE IT ARCHITECTURE}

The design of the IT Architecture is based on the "neutral" model presented (for the supply data) in par. 3 . The IT system supports a "generic" industrial cluster, consisting of a contractor and its suppliers, while exchanging information flows on supply data and (not discussed in this paper) technical and quality documentation by means of its "neutral" data base ("neutral" DBMS in Figure 6). The "neutral" data base is proposed as a viable solution in order to handle the "heterogeneity" of tools and local business operations, i.e. to archive in a "neutral" format the information generated by each cluster member.

To meet "customization" needs, specific site views (using data "views" specific for each single company of a "custom" cluster) have been developed. These views are generated via a dynamic configuration to build "customized data views" and "customized function views". Correspondingly, each contractor and each supplier views different subset of the overall data model. For example, the contractor views supply data concerning all his suppliers, whereas a specific supplier views only his supply data. Besides, each company views different sets of functions in his views of the web site, for example, the contractor views the update function for product data 
(code, description, ...) whereas a specific supplier views only the read function for product data and the update function for his inventory data.

Figure 6 presents the general design of the IT system architecture, based on the Internet technologies. "Customization" requirements are implemented in the Application Layer and in the Data Layer:

" the business logic of the application server implements the "generic" workflow and the "customized" function views;

- the data base views of the DBMS implements the "customized" data views on the "neutral" data base.

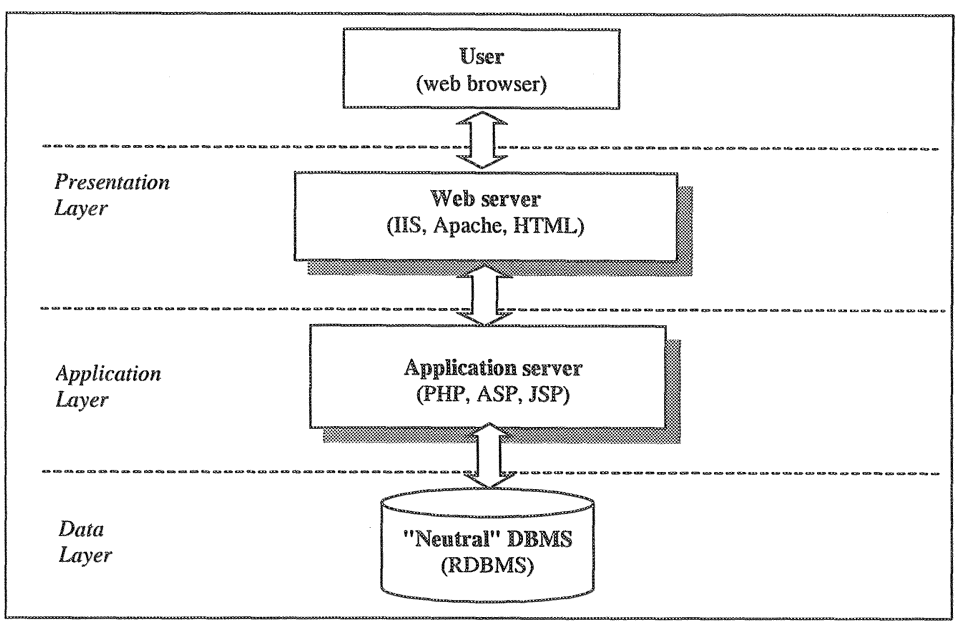

Figure 6 - IT system Architecture

Every company of a cluster can access the system via the web using its account (username and password). The account identifies the user profile, specifying if the company is a supplier or a contractor, who are its business partners (contractors or suppliers), what is its supply model (logistics rules), and so on. Based on the company profile the system generates the "site view", correspondingly with the company views:

- only the functions linked to its profile, because the business logic dynamically creates "customized" web pages;

only the data linked to its profile, because the business logic reads only the specific "customized" data views.

\section{CONCLUSIONS}

The paper discussed the main principles of an Internet-based integration of business processes for inter-enterprise collaboration, with specific concern to the "heterogeneous" context of SMEs, wherein "heterogeneous" tools enable the local operation of the business processes at each partner site. The paper then proposes the adoption of a "neutral" modelling approach as a key principle to achieve the integration of the "heterogeneity" in the Internet facility. 
This "neutral" approach is proposed by the authors as an alternative approach to the "proprietary" one, wherein "proprietary" tools are implemented to integrate a network of enterprises only according to the main contractor SW system. The "neutral" approach is proposed as an alternative one to the Internet "proprietary" solutions offered by big SW vendors for similar applications (Oracle, SAP, Baan). In the authors' opinion, such "proprietary" solutions are difficult to be implemented when the network is formed of SMEs. In fact, medium-sized enterprises (as contractors of the supply chain) may have not sufficient contractual power to impose the adoption of their "proprietary" tools to other network partners. This is also closely related to the fact that the "proprietary" solutions are typically oversized for small-sized enterprises both for the supported functions and for required investment costs. Eventually, the adoption of "proprietary" solutions may prevent small-sized enterprises from autonomy on the market, reducing their operation flexibility (one of their most important competitive factors).

Starting from the "neutral" model proposed for integration the paper also introduces the adoption of a mechanism for dynamic configuration of the Internet site views operated by each business partner. This aims to prevent from a "generic" operation of the Internet facility - that is far beyond "customization" requirements arising from small-sized enterprises.

The design of the Business and IT architecture has been validated in two clusters of SMEs of the electronic sector. The two clusters are actually experimenting the adoption of the IT system prototype, further extension of the system to the mechanical sector is planned. The experimentation aims to evaluate the effectiveness of the IT architectural solution.

An overview of the main benefits of the cooperative platform can be, any how, outlined with the actual results of the experimentation. The main benefits derive from the establishment of Internet-based relationships over standardized and more frequent information exchanges amongst contractors and suppliers.

- Standardization is expected to improve work flows in the daily operations. Actually, in fact, plenty of information media (printed papers exchanged by means of fax and files exchanged by means of Email) are adopted to exchange supply data and technical/quality documentation within a cluster. This actually leads to redundant actions (e.g., data entry from printed papers to electronic documents), potentially error-prone. Besides, due to the "heterogeneity" in information archives, consultation might result in time-consuming search activities. This is especially true for technical/quality documentation archives.

- The improvement of the information exchange and, in particular, of the consultation activities, achieved using the Internet as a unique information medium, appear to be particularly significant for small enterprises, thus helping them in "non-valued added" activities.

- The higher frequency of information exchange will facilitate reduction of the communication latency. As resulted from users' interviews, this might be particularly significant to communicate sudden changes requiring eventual reactions (such as, e.g., rush orders). Any how, an higher frequency of information exchange will help to improve, in general, synchronization in the supply chain management (e.g., in the first cluster, the already mentioned communication of in-transit material stocked at supplier site helps the 
contractor monitor "continuously" the material flows from the supplier itself and, thus, improve synchronization).

Beside assessing some benefits, the actual results of the experimentation helped also to outline some necessary enabling conditions for the adoption of the Internet facility.

" The "cluster view", already discussed throughout the paper, is an enabling condition in itself. As said, a "cluster view" was required to facilitate "graceful" adoption of the Internet facility during the daily operations, and this was mostly true for the small-sized suppliers.

" To this end, a cluster-specific "site view" was the adopted solution. This allows, on cluster features' basis, a proper allocation of functions and web resident data views. Also, this permits to develop a unique reference model of the site, i.e., a "super" model of functions and web resident data (proper for a "generic" cluster) and comprehensive of possible cluster-specific "site view" configurations. Thus, whilst information and function models are shared in the common reference model, and thus constraint possible web site configurations, the building of cluster-specific "site views" introduces web site configuration flexibility, thus helping to answer needs from specific clusters.

- Besides, other enabling conditions were also required to achieve the real adoption of the Internet facility. In particular, an efficient and reliable information transfer through the Internet was obviously requested.

- Referring to the consultation function, this was achieved mostly by means of optimized database design (throughout data base normalization methods [3]) and data visualization (throughout an hyper-textual structure of dynamic web pages designed with the UML method [7]). Referring to information upload, two upload methods have been provided to deal with different needs in terms of information frequency and volume transfer: either single item uploads, via configured masks (in case of non frequent and limited data entry activities), or, otherwise, bulk uploads, via template files structured accordingly with the information model resident in the web.

\section{ACKNOWLEDGEMENTS}

The paper results from an actually on-going project which aims to develop an Internet portal to support collaborative business processes amongst SMEs. The project is funded by L.R. $17 / 90$ on the agreement between UnionCamere Lombardia and Regione Lombardia as an initiative to improve the competitiveness of SMEs particularly craft firms - throughout Business to Business collaboration processes. Project Partners are Consorzio Politecnico Innovazione, responsible for project management; Politecnico di Milano-Dipartimento di Ingegneria Gestionale (DIG), responsible for business requirements analysis and business model design; EBLab (an integrated service centre for e-business), responsible for the design and development of the software system. 


\section{REFERENCES}

[1] Alberini S., Chiarvesio M., Grandinetti R, Pilotti L., Rutigliano M., Sommaria C. Artigianato e sistema locale. Il ruolo dei servizi nel vantaggio competitivo delle imprese artigiane. Franco Angeli, 1999.

[2] Assinform. Rapporto sull'Informatica e le Telecomunicazioni. Assinform report, 2000.

[3] Atzeni P., Ceri S., Paraboschi S., Torlone R. Database Systems: Concepts, Languages, Architectures. McGraw-Hill Education - Europe, 1999.

[4] Caroli M.G., Fratocchi L. Nuove tendenze nelle strategie di internazionalizzazione delle imprese minori. Le modalità di entrata emergenti tra alleanze e commercio elettronico. Franco Angeli, 2000.

[5] Davis R. Business Process Modelling with ARIS - A Practical Guide. Springer Verlang, London, 2001.

[6] Micelli S., Di Maria E. Distretti industriali e tecnologie di rete: progettare la convergenza. Franco Angeli, 2000.

[7] Oestereich B. Developing Software with UML: Object-oriented Analysis and Design in Practice. Addison Wesley, 1999.

[8] Scheer, A.W. ARIS - Business Process Modelling. Springer-Verlag, Berlin, 1999.

[9] Stadtler H., Kilger C. Supply Chain Management and Advanced Planning - Concepts, models, software and case studies. Springer, 2000.

[10] Voss, C.A. Developing an eService Strategy. Business Strategy Review, vol. 11, 1, 2000 , pp. $21-33$. 